\title{
A computer simulation scenario analysis approach as a decision support tool for transportation systems planning
}

\author{
G. Papageorgiou ${ }^{1}$, P. Damianou ${ }^{1}$, A. Pitsillides ${ }^{1}$, T. Aphamis ${ }^{2}$ \\ \& P. Ioannou ${ }^{3}$ \\ ${ }^{1}$ University of Cyprus, Department of Mathematics and Statistics, Cyprus \\ ${ }^{2}$ Ministry of Communication and Works, Cyprus \\ ${ }^{3}$ University of Southern California, \\ Center for Advanced Transportation Technologies, CA, USA
}

\begin{abstract}
As the level of traffic congestion rises and the complexity of traffic networks increases, the development of effective transportation strategies becomes the most challenging task for every transport authority. Meanwhile, advancements in computer technology and especially the technique of computer simulation offer a new effective way for testing plausible solutions prior to their implementation. Based on a microscopic traffic simulation model this paper presents an evaluation of the effectiveness of alternative traffic solution interventions on a main arterial in Nicosia, Cyprus. In particular, computer experiments are carried out to test various plausible scenario solutions. The scenarios under evaluation, involve the use of dedicated bus lanes, high occupancy vehicle lanes and road enhancement interventions. The simulation results show that an attractive scenario such as the use of dedicated bus lanes, if not designed properly, could bring disastrous results not only to the private car transport mode but also to the bus transport mode as coupling effects cause buses to get stuck with the rest of the traffic. By going through a computer experimentation process and scenario analysis a viable solution is derived, where restricted lanes for buses and high occupancy vehicles are introduced in combination with road geometry redesign and traffic re-direction at key points.
\end{abstract}

Keywords: traffic modelling, traffic simulation, bus priority systems, transportation management, scenario analysis. 


\section{Introduction}

In 2007 there were more than 500,000 registered vehicles in Cyprus, a figure that approaches the number of people of the island. As a result, travel times are increasing and traffic congestion becomes an everyday reality. On a daily basis in Cyprus, we are confronted with rush hours, road accidents, air pollution and driver-stress, causing an increasing number of economic, social and environmental problems.

Some of the causes of the current traffic congestion situation include the rapid economic development in Cyprus as well as the concentration of population in urban communities. Further, people are turning away from the bus and use their own private car for their daily transportation. In fact during the last three decades the number of registered vehicles has increased from 100,000 in 1980 to more than 500,000 by the year 2007 , which represent more than a $500 \%$ increase in 20 years. In addition to the increased number of cars, the use of the bus transport mode has sharply fallen. From 13 million passengers during the year 1981 we are down to 3 million for the year 2006. This represents more than a $400 \%$ decrease in the bus transport use. As a result, Cyprus cities have serious traffic congestion problems in main arterials, and at signalized intersections.

In an attempt to resolve the rising traffic congestion problem the Government of Cyprus and particularly the Ministry of Communications and Works aim for a systemic transport policy. The policy involves restraining the use of private cars, the enhancement of the urban bus transport system and betterment of its level of service, the promotion of alternative means of transport such as the bicycle, and the construction of a modern urban road network. Under this framework, specific strategies for improving the effectiveness of Cyprus traffic networks need to be developed and evaluated prior to their implementation.

In this paper such strategies are developed and tested via scenario analysis in a computer simulated environment. Specifically various scenarios are developed and tested for enhancing the quality of service of the bus transport mode as a long term solution to the traffic congestion problem. The scenario analysis is carried out via computer experiments using a traffic simulation model of an urban traffic network. The work presented in this paper is part of the Trafbus research project, partially funded by the Cyprus Research Promotion Foundation. The Trafbus research project is concerned with the modelling, simulation and analysis of traffic flow and the use of Bus Rapid Transit [1, 2] Systems in Cyprus.

Followed by a short overview on traffic flow modelling and simulation section 3 traces the development of a traffic simulation model of Strovolos Avenue, which is a main transport artery of Metropolitan Nicosia. Further, in section 3, various scenarios involving bus dedicated and high occupancy vehicle lanes are evaluated prior to their implementation. Strovolos Ave, which is depicted in figure 1, as the main road connecting the South-West to the North, exhibits the highest traffic flows as compared with the other regions of Nicosia. Further, Strovolos Avenue serves as the connector between Nicosia and a large and heavily populated area of urban and rural communities. 


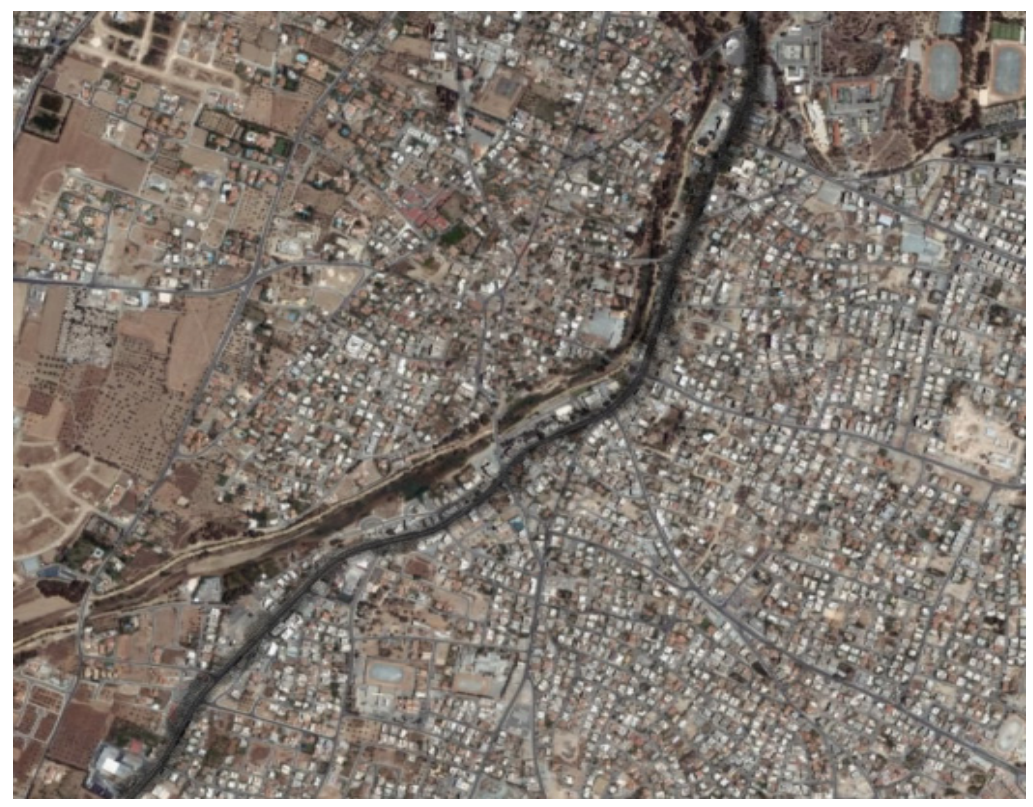

Figure 1: Strovolos Ave. with the nearby traffic network.

\section{Traffic flow modelling and simulation}

The study of traffic flow [3], and in particular vehicular traffic flow, is carried out with the aim of understanding and assisting in the prevention and remedy of traffic congestion problems. The first attempts to develop a mathematical theory for traffic flow date back to the 1930s [4,5], but even until today we do not have a satisfactory and general theory to describe real traffic flow conditions. This is because traffic phenomena are complex and nonlinear, depending on the interactions of a large number of vehicles. Moreover, vehicles do not interact simply by following the laws of physics, but are also influenced by the psychological reactions of human drivers. As a result we observe chaotic phenomena such as cluster formation and backward propagating shockwaves of vehicle speed/density [6] that are difficult if at all possible to be accurately described with mathematical models. According to a state of the art report of the Transportation Research Board [7], mathematical models for traffic flow may be classified as: Traffic Stream Characteristics Models [8], Human Factor Models [9], Car Following Models [10], Continuum Flow Models [11], Macroscopic Flow Models [12], Traffic Impact Models [13], Unsignalized Intersection Models [14], Signalized Intersection Models [15]. These models are combined in simulation software packages, which take the form of Traffic Simulation Models [16].

As a result of advancements in computer technology in recent years, the trend today is towards employing microscopic scale traffic simulation modelers in transportation studies $[17,18]$. These incorporate human factors and car 
following models in a driver-vehicle simulated based environment. Among the most widely used traffic simulation software modelers include Corsim, Vissim, Paramics and Aimsun. A comprehensive review of simulation models of traffic flow was conducted by the Institute for Transport Studies at the University of Leeds as part of the SMARTEST Project a collaborative project to develop micro-simulation tools to help solve road traffic management problems. The study compared the capabilities of more than 50 simulation packages. The results are available on the internet at http://www.its.leeds.ac.uk/projects/smartest. In our case we are utilizing Vissim [19] which is a microscopic modeler based on a psychophysical car-following model developed by Wiedemann [20].

\section{Traffic modelling and simulation of Strovolos Avenue}

As described in the previous section traffic phenomena constitute a dynamical problem situation, which make traffic modeling, and simulation a very complex, iterative and tedious process. In order to increase our chances for a successful simulation model the following methodology is adopted, which is based on the suggestions of Lieberman and Rathi [16]. This is applied for modeling the Strovolos Avenue traffic network as described below.

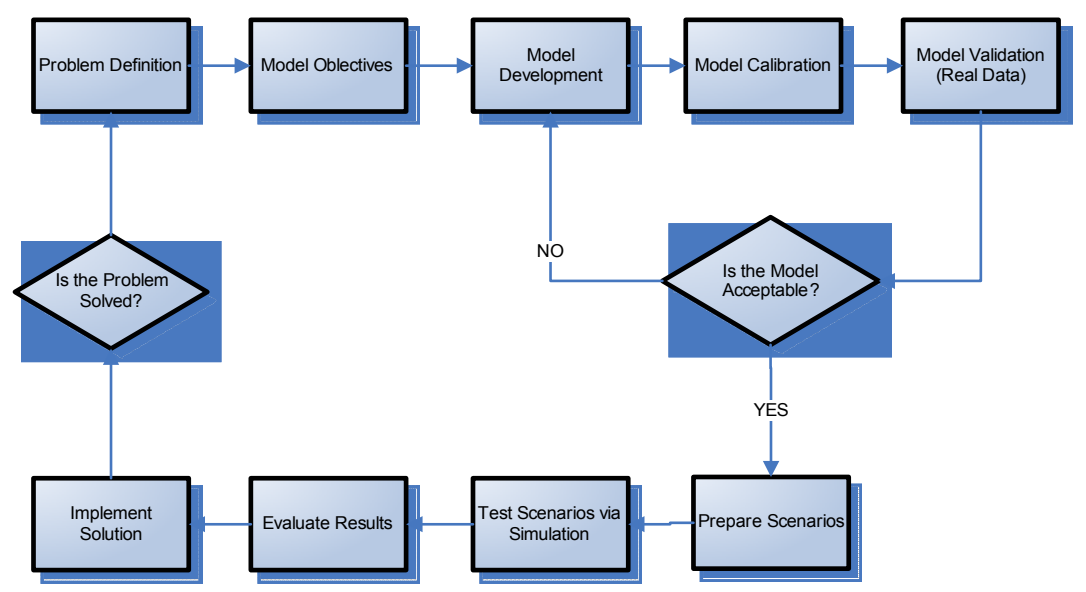

Figure 2: $\quad$ The proposed traffic modeling and simulation method.

As shown in Figure 2, the first step of the proposed approach is to identify and define the problem. In our case the symptoms of the problem that are attributed to traffic congestion manifest themselves as increasing travel times.

As explained in the introductory section the main causes for the problem of traffic congestion in Nicosia consist of the increasing number of vehicles and the decreasing use of the bus transportation system. Adding more capacity to the road infrastructure will only make things worse, as a reinforcing feedback loop is created where we encourage the further use of private vehicles and discourage 
the use of the public transport. Therefore the long term solution to the problem is to balance or even turn around the situation by encouraging the use of the public transport mode.

The question then becomes how do we change our bus transportation system and make it more attractive. This is what we aim to investigate in the Trafbus project concentrating on providing a faster and better quality level of service for our bus passengers. The objective therefore in our modeling and simulation method is to examine various scenarios such as dedicated bus lanes and Bus Rapid Transit Systems that would provide a better level of service for the bus transportation system. Meanwhile, we need to anticipate and assess any side effects of to the rest of the transportation system.

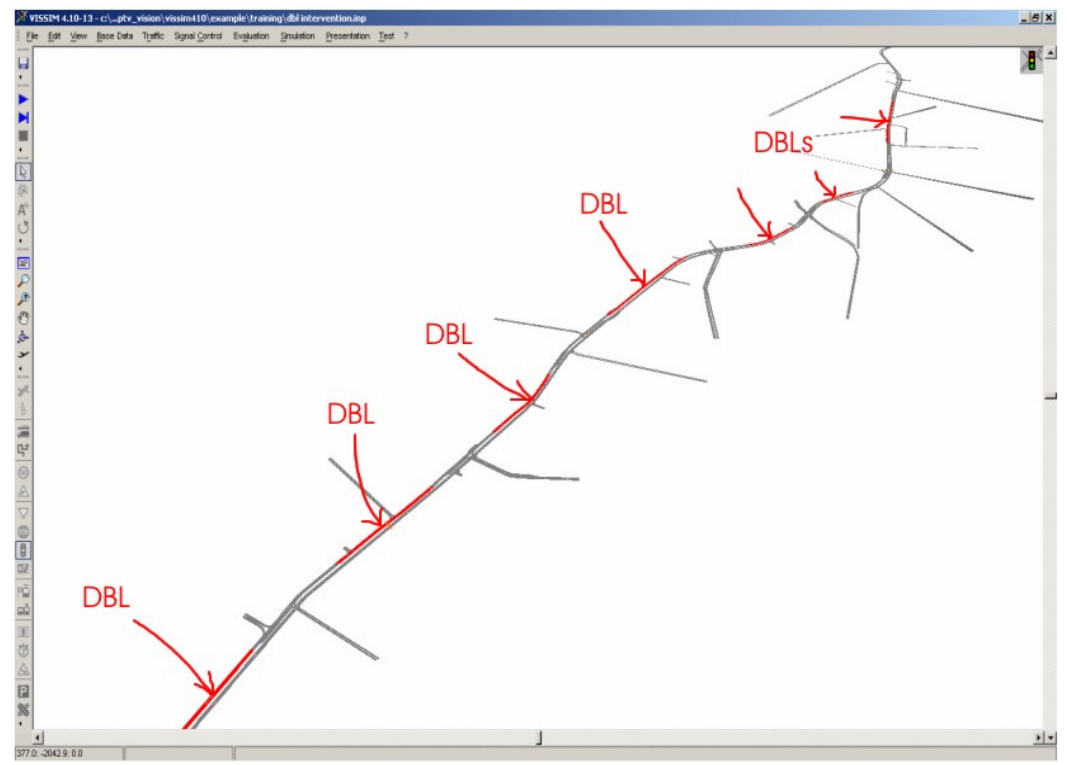

Figure 3: The simulation model of Strovolos Avenue traffic network.

Based on the stated model objectives, a simulation model of Strovolos Avenue is developed. Like any other traffic network, Strovolos Avenue consists of many traffic parameters that need to be taken into account. These include traffic control signals, priority rules, routing decisions, pedestrian crossings, signalized and unsignalised intersections and so on. A helicopter view of Strovolos Avenue simulation model, which is about 4 kilometers, two-lane road is depicted in Figure 3 (see also figure 1). Figure 3 also shows potential areas for introducing dedicated bus lanes as suggested by external consultants to the Ministry of Communications and Works. Note that under this scenario, which examined further on in this section, the one of the two lanes is given exclusively to the bus. 
Having introduced the necessary traffic parameters in our model, we enter the iterative process, which consists of model development calibration and validation of the model. Going through several iterations in developing the model, we are in a position to present some optimistic results concerning the validity of our model [21].

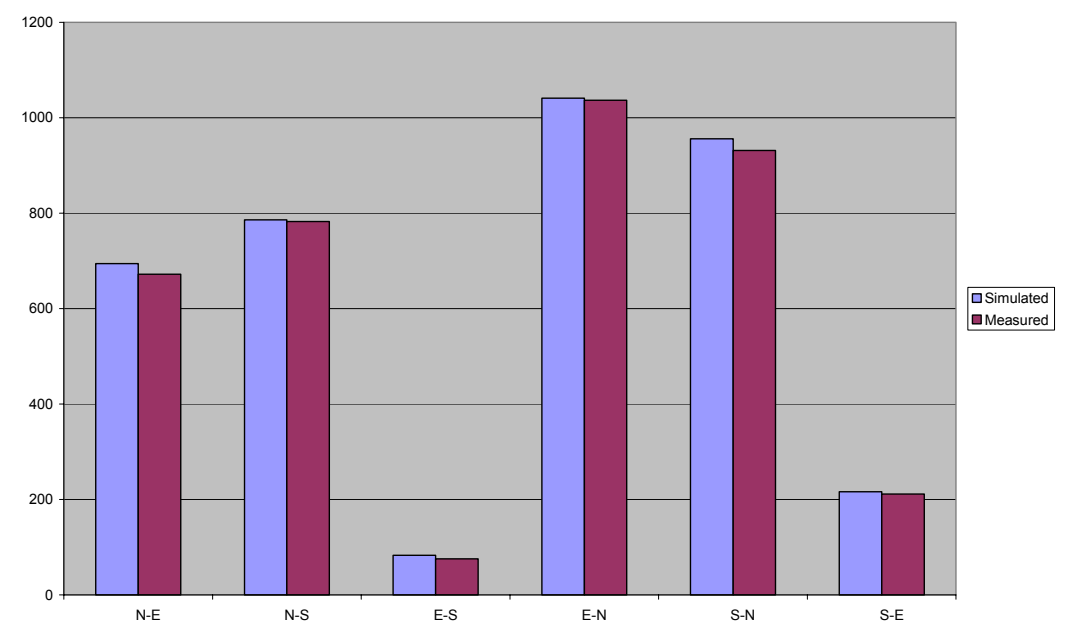

Figure 4: $\quad$ Model validation.

Figure 4 shows the real Vs simulated traffic flows of the various vehicle movement directions of a central intersection of our traffic network, that of Athalassas-Strovolou (see also figure 5). As seen in the graph below the traffic flows of real measurements obtained and those of simulated results, are quite comparable. In certain intersections such as the one shown in Figure 4, the error ranges from only $1 \%$ to $3 \%$. Further, our simulation model demonstrates the queues that we encounter in reality during the morning peak hours [22].

With a validated model in our hands, next comes the preparation of scenarios, testing and evaluation of the results. After consultations with the transportation planning section of the Ministry of Communications Works that involved suggestions from external transportation engineering consultants we came up with several plausible scenarios. In summary the various scenarios to be evaluated include the following:

1. DBL. Dedicated Bus Lanes along Strovolos Ave (as shown in Figure 3), which are restricted to all transport modes except bus use during the morning peak hours.

2. DBL-HOV. Dedicated lanes, which are restricted to be used by buses and High Occupancy Vehicles (HOV) (as shown in Figure 3) ( 2 passengers and more including the driver) during the morning peak hours 
3. DBL-HOV-Redesign. Bus-HOV dedicated lanes together with road geometry redesign and traffic re-direction where necessary depending on the simulation results.

Carrying out a series of computer experiments the performance of the network is evaluated for each of the above scenarios. Table 1 shows the simulation results and the comparison between the various scenarios and the existing situation.

Table 1: $\quad$ Scenario analysis.

\begin{tabular}{|c|c|c|c|c|c|c|c|}
\hline MOES & Base & Se-1 & 5e.2 & Se-3 & Dif- 1 & Dif. 2 & Dif -3 \\
\hline Number of vehicles in the network. HOV & 153 & 212 & 204 & 131 & $39 \%$ & $33 \%$ & $-14 \%$ \\
\hline Number of vehicles that have left the network. HOW & 1208 & 1092 & 1080 & 1236 & $-10 \%$ & $-11 \%$ & $2 \%$ \\
\hline Total Path Distance $[\mathrm{km}]$. HOV & 2226 & 1949 & 1924 & 2260 & $-12 \%$ & $-14 \%$ & $2 \%$ \\
\hline Total travel time [h]. HOV & 133 & 174 & 169 & 118 & $31 \%$ & $27 \%$ & $-11 \%$ \\
\hline Average speed [kmin]. HOW & 17 & 11 & 11 & 19 & $-33 \%$ & $-32 \%$ & $14 \%$ \\
\hline Total delay time [h]. HOV & 91 & 137 & 132 & 75 & $51 \%$ & $46 \%$ & $-17 \%$ \\
\hline Average delay time per vehiole [s]. HOV & 240 & 379 & 371 & 198 & $58 \%$ & $55 \%$ & $-18 \%$ \\
\hline Total stopped delay $[\mathrm{h}]$. HOW & 54 & 75 & 83 & 43 & $40 \%$ & $55 \%$ & $-20 \%$ \\
\hline Average stopped delay per vehicle $[s]$. HOV & 142 & 207 & 234 & 113 & $46 \%$ & $64 \%$ & $-21 \%$ \\
\hline Number of Stops. HOV & s13s & 11783 & 11242 & 6345 & $45 \%$ & $38 \%$ & $-22 \%$ \\
\hline Average number of stops per vehicles. HOV & 6 & 9 & 9 & 5 & $51 \%$ & $46 \%$ & $-22 \%$ \\
\hline Number of vehicles in the network. Car & 876 & 1241 & 1311 & sos & $42 \%$ & $50 \%$ & $-8 \%$ \\
\hline Number of vehicles that have left the network. Car & 6484 & 5821 & 5693 & 6635 & $-10 \%$ & $-12 \%$ & $2 \%$ \\
\hline Total Path Distance $[\mathrm{km}]$. Car & 12100 & 10597 & 10206 & 12149 & $-12 \%$ & $-16 \%$ & $0 \%$ \\
\hline Total travel time [h]. Car & 734 & 948 & 1015 & 665 & $29 \%$ & $38 \%$ & $-9 \%$ \\
\hline Average speed [kmth]. Car & 16 & 11 & 10 & 18 & $-32 \%$ & $-39 \%$ & $11 \%$ \\
\hline Total delay time [h]. Car & 503 & 746 & 821 & 433 & $48 \%$ & $63 \%$ & $-14 \%$ \\
\hline Average delay time per vehicle $[s]$. Car & 246 & 380 & 422 & 210 & $55 \%$ & $71 \%$ & $-15 \%$ \\
\hline Total stopped delay $[\mathrm{h}]$. Car & 308 & 411 & 494 & 238 & $34 \%$ & $60 \%$ & $-23 \%$ \\
\hline Average stopped delay per vehicle $[s]$. Car & 150 & 210 & 254 & 115 & $39 \%$ & $69 \%$ & $-23 \%$ \\
\hline Number of Stops. Car & 43168 & 63492 & 71899 & 36942 & $47 \%$ & $67 \%$ & $-14 \%$ \\
\hline Average number of stops per vehicles. Car & $\epsilon$ & 9 & 10 & 5 & $53 \%$ & $75 \%$ & $-15 \%$ \\
\hline Number of vehicles in the network. Bus & 2 & 7 & 7 & 3 & $250 \%$ & $250 \%$ & $50 \%$ \\
\hline Number of vehicles that have left the network. Bus & 21 & 16 & 16 & 20 & $-24 \%$ & $-24 \%$ & $-5 \%$ \\
\hline Total Path Distance $[\mathrm{km}]$. Bus & 48 & 37 & 38 & 48 & $-22 \%$ & $-20 \%$ & $\alpha \%$ \\
\hline Total travel time [h]. Bus & 5 & 6 & 6 & 4 & $36 \%$ & $39 \%$ & $-22 \%$ \\
\hline Average speed [kmth]. Bus & 10 & 6 & 6 & 13 & $-43 \%$ & $-43 \%$ & $28 \%$ \\
\hline Total delay time $[\mathrm{h}]$. Bus & 3 & 5 & 5 & 2 & $58 \%$ & $60 \%$ & $-30 \%$ \\
\hline Average delay time per vehicle $[s]$. Bus & 528 & 833 & 845 & 371 & $58 \%$ & $60 \%$ & $-30 \%$ \\
\hline Total stopped delay [h]. Bus & 2 & 3 & 3 & 1 & $92 \%$ & $95 \%$ & $-33 \%$ \\
\hline Average stopped delay per vehiole [s]. Bus & 254 & 488 & 496 & 170 & $92 \%$ & $95 \%$ & $-33 \%$ \\
\hline Number of Stops. Bus & 353 & 409 & 484 & 234 & $16 \%$ & $37 \%$ & $-34 \%$ \\
\hline Average number of stops per vehicles. Bus & 15 & 18 & 21 & 10 & $16 \%$ & $37 \%$ & $-34 \%$ \\
\hline
\end{tabular}

Specifically on table 1 we see various measures of effectiveness for the whole simulated traffic network based on the existing situation and the three plausible scenario solutions, that is dedicated bus lanes, dedicated bus lanes and high occupancy vehicle lanes, and the latter plus interventions on the road geometry and traffic flow. The measures of effectiveness include the number of vehicles in the network, the total path distance traveled, the total travel time, the average speed, the total delay time, the average delay time per vehicle, the total stopped delay, the average stopped delay, the total number of stops and the average number of stops, for the HOV, private car and bus transport modes.

As it can be observed from table 1, the dedicated bus lane scenario, which seems to be an attractive solution for enhancing the quality of service of the bus transport mode, proves to be a disaster. The simulation results show that for the bus transport mode total travel time is increased by $36 \%$, average speed is decreased by $43 \%$ and delay time is increased by $58 \%$. As expected the quality of service for the private car transport mode also deteriorates as shown in the simulation results. This is may be attributed obviously to the loss of capacity but also to the coupling effects and the high complexity of the traffic network. 
In the scenario of dedicated lanes for both the bus transport mode and high occupancy vehicles the situation gets slightly better but still is a lot worse than the existing situation for all transport modes as shown on Table 1. Simulation results of network performance for the bus transport mode show still an increase in travel time by $39 \%$, a drop in the average speed by $43 \%$, and an increase in delay time by $60 \%$.

The above results clearly demonstrate that an intuitively attractive alternative could be disastrous when it is not properly designed. From the visualization of the simulation runs it becomes clear that a bottleneck is created at the intersection between Athalassas Ave and Strovolos Ave (see Figure 5). At this intersection traffic flow is directed towards the centre and since a long restricted lane before the intersection reduces the capacity of the road from two lanes to one lane and since also there is no exclusive right turn movement at the junction going eastwards high congestion is created. This directed our thinking towards redesigning the traffic network under the constraint of not being able to add a third lane exclusively for buses.

Redesigning the road geometry by adding an exclusive right turn link to the current two-lane road, gave us no real improvement. But by redesigning the exclusive right-turn as an underpass or another way so that the vehicles they want to turn right towards Athalassas Ave. do that relatively freely then the simulation results show some quite promising improvements on the various measures of effectiveness of the traffic network.

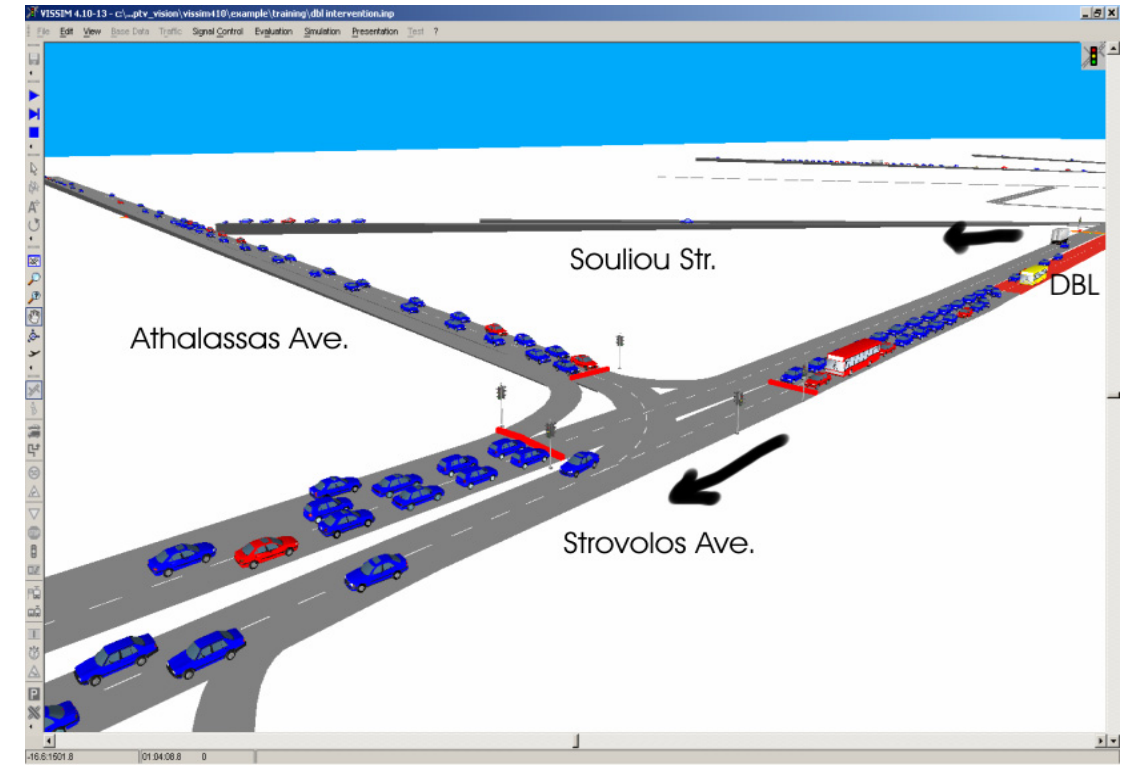

Figure 5: The dedicated bus and high occupancy vehicle lane with road redesign and traffic re-direction Scenario - (Strovolos /Athalassas intersection). 
As we can observe from Table 1, there are significant improvements for buses and high occupancy vehicles as well as for single occupancy vehicles. Especially for the bus transport mode travel times reduce by $22 \%$ average speed increases by $28 \%$ and average delay decreases by $30 \%$. These results would be valid provided that a new traffic bottleneck is not created downstream especially at the roundabout of the Presidential Palace, something that should be investigated before anything is implemented. Further, safety issues need to be assessed for any redesign.

Figure 5 shows a screenshot of the simulated model of the DBL-HOVredesign scenario where the bottleneck at Athalassas Ave. intersection is removed. As a simple practical alternative design Souliou str. (shown in Figure 4 as the street in the background joining the two main arterials of Strovolos and Athalassas Ave.) is utilized as a drive through road for the vehicles that want to turn right towards Athalassas Ave. This is by no means a suggestion that it constitutes the best alternative, as traffic congestion is transferred to two new intersections, and further safety issues are raised. An investigation is necessary to test other ways of redirecting traffic towards Athalassas Ave, which through scenario analysis was found to be the main bottleneck of the traffic network. As mentioned above a plausible solution that emerges is to construct an underpass for exclusive use by the traffic turning right towards Athalassas Ave.

Finally, we foresee that through the DBL-HOV-Redesign solution the increase of the quality of service of the bus transport mode will encourage more people to switch from the private car to the bus transport mode and thereby traffic flows will decrease. Further, with the DBL-HOV-Redesign alternative, the current high occupancy vehicles, which are considered to be vehicles carrying two or more passengers, would encourage more commuters to drive together and thereby the number of vehicles on the road will decrease and the level of traffic congestion will eventually be reduced or at least stabilize.

\section{Conclusions}

Computer simulation proves to be a very powerful tool for analyzing complex dynamical problems such as traffic congestions. On the other hand, the development of computer simulation models requires extensive time, effort, rigour and high persistence. In this paper an approach to modeling and simulating traffic networks is proposed and implemented. The proposed approach goes through various stages, which include problem identification, model objectives, model development, model calibration, model validation, scenario preparation, simulation experiments and simulated results evaluation. The proposed approach is applied in the case of developing a microscopic traffic simulation model for an urban traffic network in Strovolos, Nicosia, Cyprus.

Utilizing the microscopic simulation model, various scenarios, which take the form of computer experiments, were carried out. As seen from the simulation results the intuitively attractive scenario of bus dedicated lanes could make things worse if not properly designed and any side effects are not anticipated. A better scenario but still a lot worse than the existing for all transport modes with respect measures of effectiveness related to travel time and time delays is the 
combined high occupancy vehicle and bus dedicated lanes. On the contrary, through proper analysis and computer simulation experimentation a viable solution is derived where the measures of effectiveness of travel time, average speed and time delay show significant improvements. Further scenario solutions we are planning to evaluate, involve interventions on the signal traffic controllers with signal pre-emption, synchronization and extra traffic lights for bus advance. We are actually now in the process of testing these new scenarios in our computer traffic simulated environment.

\section{References}

[1] Transit Cooperative Research Program, TRCP Report 90, Bus Rapid Transit, TRB, Washington D.C 2003, www.TRB.org

[2] "BRT-Bus Rapid Transit-Why More Communities are Choosing Bus Rapid Transit" TRB, Washington D.C 2003

[3] A. May, Traffic Flow Fundamentals, Prentice Hall, 1990.

[4] B.D. Greenshields, "A Study in Highway Capacity", Highway Research Board, Proceedings, Vol. 14, 1935, p. 458.

[5] W.F. Adams, "Road Traffic Considered as a Random Series", J. Inst. Civil Engineers, 4, 1936, pp. 121-130.

[6] A. Bose, and P. Ioannou, "Shock Waves in Mixed Traffic Flow", IFAC Symposium on Control in Transportation Systems, 2000.

[7] N.H. Gartner, C. Messer, A.K. Rathi, "Introduction in Traffic Flow Theory", Washington, D.C.: US Federal Highway Administration, 1996, pp. 1-1 to $1-4$

[8] F.L. Hall, "Traffic stream characteristics in Traffic Flow Theory", Washington, D.C.: US Federal Highway Administration, 1996, pp. 2-1 to 2-34

[9] R.J. Koppa, "Human Factors" in Traffic Flow Theory, Washington, D.C.: US Federal Highway Administration, 1996, pp. 3-1.

[10] R.W. Rothery, "Car Following Models in Traffic Flow Theory", Washington, D.C.: US Federal Highway Administration, 1996, pp. 4-1-442.

[11] R. Kuhne, and P. Michalopoulos, "Continuum Flow Models in Traffic Flow Theory”, Washington, D.C.: US Federal Highway Administration, 1996, pp. 5-1-5-51

[12] J.C. Williams, "Macroscopic Flow Models in Traffic Flow Theory", Washington, D.C.: US Federal Highway Administration, 1996, pp. 6-1-331.

[13] S. Ardekani, E. Hauerand and E. Jamei, "Traffic Impact Models in Traffic Flow Theory", Washington, D.C.: US Federal Highway Administration, 1996, pp. 7-1-7-24.

[14] R.J. Troutbeck, and W. Brilon "Unsignalized Intersection Theory in Traffic Flow Theory", Washington, D.C.: US Federal Highway Administration, 1996, pp. 8-1-8-47. 
[15] N. Rouphail, A. Tarko and J. Li, "Traffic Flow at Signalized Intersections in Traffic Flow Theory", Washington, D.C.: US Federal Highway Administration, 1996, pp. 9-1-9-32.

[16] E. Lieberman, and A.J. Rathi 1996, "Traffic Simulation in Traffic Flow Theory", Washington, D.C.: US Federal Highway Administration, pp. 10$1-3-23$.

[17] Minh et al. 'Traffic Policy Evaluations Using Micro Traffic Simulation: A case Study of Thailand' Transportation Research Board Annual Meeting, January 2003, pp1-22

[18] Mirchandani et al ' Traffic Assignment Using Iterated Route-Based Simulation' Transportation Research Board Annual Meeting, January 2003, pp1-16

[19] M. Fellendorf, and P. Vortisch, "Validation of the Microscopic Traffic Flow Model VISSIM in Different Real-World Situations," Paper presented at the Annual Meeting, TRB, Washington, DC, 2001

[20] R. Wiedemann, „Simulation des Straßenverkehrsflusses. Schriftenreihe des Instuts für Verkehrswesen der Universität Karlsruhe“, 1974, Heft 8.

[21] G. Papageorgiou, Towards a microscopic simulation model for traffic management: a computer based approach 11th IFAC Symposium on Control in Transportation Systems, 2006, Netherlands.

[22] G. Papageorgiou, P. Damianou, A. Pitsillides, T. Aphames, P. Ioannou, A Microscopic Traffic Simulation Model for Transportation Planning in Cyprus, International Conference on Intelligent Systems And Computing: Theory And Applications (ISYC), 2006, Cyprus 